\title{
Matrix continued fraction expansion of Bessel function
}

\author{
Ali Kacha, Gul Karadeniz Gozeri ${ }^{1}$ and Kacem Belhroukia
}

Amgnca Laboratory, Faculty of Science, Ibn Tofail University, Kenitra, Morocco

${ }^{1}$ Department of Mathematics, Faculty of Science, Istanbul University, Istanbul, Turkiye

Received: 22 October 2015, Accepted: 8 March 2016

Published online: 19 June 2016.

\begin{abstract}
The aim of this paper is to provide some results and applications of continued fractions with matrix arguments. First, we recall some properties of matrix functions with real coefficients. Afterwards, we give a matrix continued fraction expansion of the Bessel function.
\end{abstract}

Keywords: Continued fraction expansion, positive definite matrix, matrix continued fraction, development of series, Bessel function.

\section{Introduction and motivation}

The theory of continued fractions has been a topic of great interest over the last two hundred years. The basic idea of this theory over real numbers is to give an approximation of various real numbers by the rational ones. One of the main reasons why continued fraction are so useful in computation is that they often provide representation for transcendental function that are much more generally valid than the classical representation by, say, the power series. Further; in the convergent case, the continued fractions expansions have the advantage that they converge more rapidly than other numerical algorithms. In Banach spaces, generalizations of some results of real cases are published by Haydan [5] and Negoescu [9].

Recently, the extension of continued fractions theory from real numbers to the matrix case has seen several developments and interesting applications (see [2],[4],[7],[9]). The real case is relatively well studied in the literature. However, in contrast to the theoretical importance, one can find in mathematical literature only a few results on the continued fractions with matrix arguments. There have been some reasons why all this attention has been devoted to what is, in essence, a very humble idea. Since calculations involving matrix valued functions with matrix arguments are feasible with large computers, it will be an interesting attempt to develop such matrix theory. The main difficulty arises from the fact that the algebra of square matrices is not commutative.

For simplicity and clearness, we restrict ourselves to positive definite matrices, but our results can be, without special difficulties, projected to the case of positive definite operators from an infinite dimensional Hilbert space into itself.

Bessel functions occupy a very important place in the problems solutions with cylindrical symmetry. We can say that they are important in the hierarchy of functions which are necessaries to tabulate. Bessel functions come immediately after the trigonometric functions, logarithm and exponential function but it is customary to fit them in the class of so-called special functions. There are many books deal with remarkable properties of these functions. 
This article is organized as follows: The section 2 contains some basic notions and results about matrix continued fractions that are needed later. In Section 3, we give a continued fractions expansion of the Bessel function $J_{n}(A)$, for $n \geq 1$ of a positive definite matrix A.

\section{Preliminary and notations}

The functions of matrix arguments play a widespreased role in science and engineering, with applications areas ranging from nuclear magnetic resonance [1]. So for any scalar polynomial $p(z)=\sum_{i=0}^{k} c_{i} i_{z}^{i}$ gives rise to a matrix polynomial with scalar coefficients by simply substituting $A^{i}$ for $z^{i}$ :

$$
P(A)=\sum_{i=0}^{k} c_{i} A^{i}
$$

More generally, for a function $f$ with a series representation on an open disk containing the eigenvalues of $A$, we are able to define the matrix function $f(A)$ via the Taylor series for $f[8]$.

Alternatively, given a function $f$ that is analytic inside a closed contour $\Gamma$ which encloses the eigenvalues of $A, f(A)$ can be defined, by analogy with Cauchy's integral theorem by

$$
f(A)=\frac{1}{2 \pi i} \int_{\Gamma} f(z)(z I-A)^{-1} d z .
$$

The definition is known as the matrix version of Cauchy's integral theorem. Let $\mathscr{M}_{m}$ be the algebra of real square matrices, we now mention an important result of matrix functions.

Lemma 1. Let $f$ be an analytic function in a domain $D$.

(i) If two matrices $A \in \mathscr{M}_{m}$ and $B \in \mathscr{M}_{m}$ are similar, with $A=Z B Z^{-1}$, and $s p(A) \subset D$, then the matrices $f(A)$ and $f(B)$ are also similar, with $f(A)=Z f(B) Z^{-1}$.

(ii) ) If $A \in \mathscr{M}_{m}$ is a block diagonal matrix $A=\operatorname{diag}\left(A_{1}, A_{2}, \ldots, A_{r}\right)$ then $f(A)=\operatorname{diag}\left(f\left(A_{1}\right), f\left(A_{2}\right), \ldots, f\left(A_{r}\right)\right)$.

Proof.

(i) For $A=Z B Z^{-1}$ we have $A^{k}=Z B^{k} Z^{-1}$. Hence for every polynomial $p(Z)$ it follows that

$$
p(A)=Z p(B) Z^{-1}
$$

Therefore if either one of $p(A)$ or $p(B)$ equals zero then so does the other, implying that $A$ and $B$ share the same minimal polynomial. From definition there exists an interpolating polynomial $r(Z)$ such that

$$
f(A)=r(A), f(B)=r(B)
$$

and since for every polynomial we have $p(A)=Z p(B) Z^{-1}$, the result follows.

(ii) We deduce it from (i).

Let $A \in \mathscr{M}_{m}, A$ is said to be positive semidefinite (resp. positive definite) if $A$ is symmetric and

$$
\forall x \in \mathbb{R}^{m},<A x, x>\geq 0 \quad\left(\text { resp. } \forall x \in \mathbb{R}^{m}, x \neq 0<A x, x>>0\right)
$$


where $<., .>$ denotes the standard scalar product of $\mathbb{R}^{m}$.

We observe that positive semidefiniteness induces a partial ordering on the space of symmetric matrices: if $A$ and $B$ are two symmetric matrices, then we write $A \leq B$ if $B-A$ is positive semidefinite.

Henceforth, whenever we say that $A \in \mathscr{M}_{m}$ is positive semidefinite (or positive definite), it will be assumed that $A$ is symmetric.

For any matrices $A, B \in \mathscr{M}_{m}$ with $B$ invertible, we write $A / B=B^{-1} A$, in particular, if A=I, the matrix identity, then $I / B=B^{-1}$. It is easy to verify that for any invertible matrix $\mathrm{X}$ we have

$$
\frac{A}{B}=\frac{X A}{X B} \neq \frac{A X}{B X}
$$

Definition 1. Let $\left\{A_{n}\right\}_{n \geq 0}$ and $\left\{B_{n}\right\}_{n \geq 1}$ be two sequences of matrices in $\mathscr{M}_{m}$. We denote the continued fraction expansion by

$$
A_{0}+\frac{B_{1}}{A_{1}+\frac{B_{2}}{A_{2}+\ldots}}:=\left[A_{0} ; \frac{B_{1}}{A_{1}}, \ldots, \frac{B_{n}}{A_{n}}\right] .
$$

Sometimes, we denote this continued fraction by $\left[A_{0} ; \frac{B_{n}}{A_{n}}\right]_{n=1}^{+\infty}$ or $A_{0}+K\left(B_{n} / A_{n}\right)$.

The fractions $\frac{B_{n}}{A_{n}}$ and $\frac{P_{n}}{Q_{n}}:=\left[A_{0} ; \frac{B_{i}}{A_{i}}\right]_{i=1}^{n}$ are called, respectively, the $n^{\text {th }}$ partial quotient and the $n^{\text {th }}$ convergent of the continued fraction $A_{0}+K\left(B_{n} / A_{n}\right)$.

We note that the evaluation of $n^{\text {th }}$ convergent according to the above definitions is not practical because we have to repeatedly inverse matrices. The following proposition gives an adequate method to calculate $A_{0}+K\left(B_{n} / A_{n}\right)$.

Proposition 1. [10]. For the continued fraction $A_{0}+K\left(B_{n} / A_{n}\right)$, define

$$
\left\{\begin{array} { l } 
{ P _ { - 1 } = I , P _ { 0 } = A _ { 0 } } \\
{ Q _ { - 1 } = 0 , Q _ { 0 } = I }
\end{array} \text { and } \left\{\begin{array}{c}
P_{n}=A_{n} P_{n-1}+B_{n} P_{n-2} \\
Q_{n}=A_{n} Q_{n-1}+B_{n} Q_{n-2}
\end{array} n \geq 1 .\right.\right.
$$

Then $Q_{n}^{-1} P_{n}$ is the $n^{\text {th }}$ convergent of the continued fraction $A_{0}+K\left(B_{n} / A_{n}\right)$.

The proof of the next proposition is elementary and we left it to the reader.

Proposition 2. For any two matrices $C$ and $D$ with $C$ invertible, we have

$$
C\left[A_{0} ; \frac{B_{k}}{A_{k}}\right]_{k=1}^{n} D=\left[C A_{0} D ; \frac{B_{1} D}{A_{1} C^{-1}}, \frac{B_{2} C^{-1}}{A_{2}}, \frac{B_{k}}{A_{k}}\right]_{k=3}^{n} .
$$

Definition 2. Let $\left\{A_{n}\right\},\left\{B_{n}\right\}\left\{C_{n}\right\}$ and $\left\{D_{n}\right\}$ be four sequences of matrices. We say that the continued fractions $A_{0}+$ $K\left(B_{n} / A_{n}\right)$ and $C_{0}+K\left(D_{n} / C_{n}\right)$ are equivalent if we have $F_{n}=G_{n}$ for all $n \geq 1$, where $F_{n}$ and $G_{n}$ are the $n^{\text {th }}$ convergents of $A_{0}+K\left(B_{n} / A_{n}\right)$ and $C_{0}+K\left(D_{n} / C_{n}\right)$, respectively. 
In order to simplify the statements on some partial quotients of continued fractions with matrix arguments, we need the following proposition which is an example of equivalent continued fractions.

Proposition 3. Let $\left[A_{0} ; \frac{B_{k}}{A_{k}}\right]_{k=1}^{+\infty}$ be a given continued fraction. Then

$$
\frac{P_{n}}{Q_{n}}:=\left[A_{0} ; \frac{B_{k}}{A_{k}}\right]_{k=1}^{n}=\left[A_{0} ; \frac{X_{k} B_{k} X_{k-2}^{-1}}{X_{k} A_{k} X_{k-1}^{-1}}\right]_{k=1}^{n}
$$

where $X_{-1}=X_{0}=I$ and $X_{1}, X_{2}, \ldots, X_{n}$ are arbitrary invertible matrices.

Proof. Let $\frac{P_{n}}{Q_{n}}$ and $\frac{\widetilde{P}_{n}}{\widetilde{Q}_{n}}$, be the $n^{\text {th }}$ convergents of the continued fractions $\left[A_{0} ; \frac{B_{k}}{A_{k}}\right]_{k=1}^{+\infty}$ and $\left[A_{0} ; \frac{X_{k} B_{k} X_{k-2}^{-1}}{X_{k} A_{k} X_{k-1}^{-1}}\right]_{k=1}^{+\infty}$, respectively. By proposition 2, for all $n \geq 1$, we can write

$$
\widetilde{P}_{n}=X_{n} A_{n} X_{n-1}^{-1} \widetilde{P_{n-1}}+X_{n} B_{n} X_{n-2}^{-1} \widetilde{P_{n-2}}
$$

which is equivalent to

$$
X_{n}^{-1} \widetilde{P}_{n}=A_{n}\left(X_{n-1}^{-1} \widetilde{P_{n-1}}\right)+B_{n}\left(X_{n-2}^{-1} \widetilde{P_{n-2}}\right)
$$

This last result joined to the initial conditions prove that for all $n \geq 1, X_{n}^{-1} \widetilde{P}_{n}=P_{n}$.

A similar result can be obtained for $Q_{n}$. Consequently, both continued fractions have the same convergents and the proof of proposition 3 follows. We also recall the following proposition in real case.

Proposition 4. [6]. Let $\left(r_{n}\right)$ be a non-zero sequence of real numbers. We prove easily that the following continued fractions

$$
\left[a_{0} ; \frac{b_{1}}{a_{1}}, \frac{b_{2}}{a_{2}}, \ldots, \frac{b_{n}}{a_{n}}, \ldots\right] \text { and }\left[a_{0} ; \frac{r_{1} b_{1}}{r_{1} a_{1}}, \frac{r_{2} r_{1} b_{2}}{r_{2} a_{2}}, . ., \frac{r_{n-1} r_{n} b_{n}}{r_{n} a_{n}}, \ldots\right]
$$

are equivalent.

\section{Main results}

Our aim in this section is to give the continued fraction expansions of the Bessel function $J_{n}(A)$ for all $n \in \mathbb{N}$, where A is a positive definite matrix.

\subsection{Continued fractions expansion of $J_{n}(x)$.}

For simplicity, we start with the real case and we begin by recalling some properties. The following differential equation

$$
x^{2} \frac{d^{2} F}{d x^{2}}(x)+x \frac{d F}{d x}(x)+\left(x^{2}-v^{2}\right) F(x)=0
$$

is called Bessel differential equation of order $v$ where $v$ is a real number. The solutions (see [2]) of this equation can be written

$$
F(x)=C_{1} J_{v}(x)+C_{2} Y_{v}(x)
$$


where $J_{v}$ and $Y_{v}$ are Bessel functions of order $v$ of the first and second kinds respectively which are given by:

$$
J_{v}(x)=\left(\frac{x}{2}\right)^{v} \sum_{k=0}^{\infty} \frac{(-1)^{k}\left(\frac{x}{2}\right)^{2 k}}{k ! \Gamma(v+k+1)}, \quad|\arg x|<\pi, \quad v \in \mathbb{C}
$$

where $\Gamma$ is gamma function and

$$
Y_{v}(x)=\frac{J_{v}(x) \cos (\pi v)-J_{-v}(x)}{\sin (\pi v)}, \quad|\arg x|<\pi, \quad v \in \mathbb{C} \backslash \mathbb{Z} .
$$

Let $n \in \mathbb{N}$, we define the Bessel function $J_{n}$ of index $n$ and the power series of $J_{n}(x)$ is

$$
J_{n}(x)=\left(\frac{x}{2}\right)^{n} \sum_{k=0}^{+\infty} \frac{(-1)^{k}}{k !(n+k) !}\left(\frac{x}{2}\right)^{2 k}
$$

Theorem 1. Let $n \in \mathbb{N}$, the continued fraction expansion of $J_{n}(x)$ is given by

$$
J_{n}(x)=\left[\frac{x^{n}}{2^{n} n !} ; \frac{\frac{-x^{n+2}}{2^{n+1}(n+1) !}}{1}, \frac{x^{2}}{2^{3}(n+2)-x^{2}}, \frac{(-1)^{k} x^{n+k-2}}{\alpha(n, k)\left(4 k(n+k)-x^{2}\right)}\right]_{k=3}^{+\infty},
$$

where $\alpha(n, k)=2^{n+2 k-4}(k-2) !(n+k-2) !$.

To prove the this theorem. We need the next lemma. In the following lemma, from the development of a function given by the Taylor series, we give the development in continued fractions of the series that was established by Euler.

Lemma 2. [6]. Let $f$ be a function with the Taylor serie development is $f(x)=\sum_{k=0}^{+\infty} c_{k} x^{k}$ in $D \subset \mathbb{R}$. Then, the development in continued fraction of $f(x)$ is

$$
f(x)=\sum_{k=0}^{+\infty} c_{k} x^{k}=\left[c_{0} ; \frac{c_{1} x}{1}, \frac{-c_{2} x}{c_{1}+c_{2} x}, \frac{-c_{1} c_{3} x}{c_{2}+c_{3} x}, \ldots, \frac{-c_{k-2} c_{k} x}{c_{k-1}+c_{k} x}, . .\right] .
$$

Proof. (Proof of Theorem (1)). We use Lemma (3.2) for the function:

$$
J_{n}(x)=\sum_{k=0}^{\infty}(-1)^{k} \frac{\left(\frac{x}{2}\right)^{n+2 k}}{k !(n+k) !}=\sum_{k=0}^{\infty} \frac{(-1)^{k}(x)^{n+k}}{2^{n+2 k} k !(n+k) !} x^{k}
$$

by putting

$$
C_{k}=\frac{(-1)^{k} x^{n+k}}{k !(n+k) ! 2^{n+2 k}}
$$

For $k \geq 3$, we get:

$$
C_{k-2} C_{k}=\frac{x^{2 n+2 k-2}}{4^{n+2 k-2} k !(k-2) !(n+k) !(n+k-2) !} .
$$


Furthermore, we have

$$
C_{k-1}+C_{k} x=\frac{(-1)^{k-1} x^{n+k-1}\left(4 k(n+k)-x^{2}\right)}{2^{n+2 k} k !(n+k) !}
$$

As a result,

$$
\frac{C_{k-2} C_{k}}{C_{k-1}+C_{k} x}=\frac{(-1)^{k-1} x^{n+k-1}}{2^{n+2 k-4}(k-2) !(n+k-2) !\left(4 k(n+k)-x^{2}\right)}
$$

In particular, we have

$$
C_{0}=\frac{x^{n}}{2^{n} n !}, \quad C_{1}=\frac{-x^{n+1}}{2^{n+2}(n+1) !}, \quad C_{2}=\frac{x^{n+2}}{2^{n+5}(n+2) !}, \quad \frac{C_{2} x}{C_{1}+C_{2} x}=\frac{-x^{2}}{2^{3}(n+2)-x^{2}}
$$

Therefore, the development of continued fraction of $\operatorname{Jn}(x)$ is

$$
J_{n}(x)=\left[\frac{x^{n}}{2^{n} n !} ; \frac{\frac{-x^{n+2}}{2^{n+1}(n+1) !}}{1}, \frac{x^{2}}{2^{3}(n+2)-x^{2}}, \frac{(-1)^{k} x^{n+k-1}}{\alpha(n, k)\left(4 k(n+k)-x^{2}\right)}\right]_{k=3}^{+\infty}
$$

\subsection{Continued fraction expansion of $J_{n}(A)$.}

The next theorem is a matrix version of the previous theorem 1 .

Theorem 2. Let $A$ be a positive definite matrix of $\mathscr{M}_{m}$. A continued fraction expansion of $J_{n}(A)$ is given by,

$$
J_{n}(A)=\left[\frac{A^{n}}{2^{n} n !} ; \frac{\frac{-A^{n+2}}{2^{n+1}(n+1) !}}{1}, \frac{A^{2}}{2^{3}(n+2)-A^{2}}, \frac{(-1)^{k} A^{n+k-1}}{\alpha(n, k)\left(4 k(n+k)-A^{2}\right)}\right]_{k=3}^{+\infty}
$$

Proof. (Proof of Theorem (2)). Let $A \in \mathscr{M}_{m}$ be a positive definite matrix. Then there exists an invertible matrix $X$ such that $A=X D X^{-1}$, where $D=\operatorname{diag}\left(\lambda_{1}, \lambda_{2}, \ldots, \lambda_{m}\right)$ and $\lambda_{i}>0$.

As the function $z \rightarrow J_{n}(z)$ is analytic in the open halfplane $\{z \in \mathbb{C}, \operatorname{Re}(z)>0\}$, then

$$
J_{n}(A)=X J_{n}(D) X^{-1}=X \operatorname{diag}\left(J_{n}\left(\lambda_{1}\right), J_{n}\left(\lambda_{2}\right), . ., J_{n}\left(\lambda_{m}\right)\right) X^{-1}
$$

Let us define the sequences $\left\{P_{n}\right\}$ and $\left\{Q_{n}\right\}$ by :

$$
\begin{gathered}
\left\{\begin{array}{l}
P_{-1}=I, P_{0}=\frac{D^{n}}{2^{n} n !}, P_{1}=\frac{D^{n}}{2^{n+1}(n+1) !} \\
Q_{-1}=0, Q_{0}=I, Q_{1}=I
\end{array}\right. \\
\left\{\begin{array}{l}
P_{2}=\frac{(n+2)}{2^{n}(n+1) !} D^{n}\left(1-D^{2}\right)\left((n+1)+D^{2}\right) \\
Q_{2}=\frac{1}{2^{n+1}(n+1) !}\left(2^{n+2}(n+2) ! I-2^{n+1}(n+1) ! D^{2}-D^{n+2}\right)
\end{array}\right.
\end{gathered}
$$


and for $k \geq 3$,

$$
\left\{\begin{array}{l}
P_{k}=\alpha(n, k)\left(4 k(n+k) I-D^{2}\right) P_{k-1}+(-1)^{k} D^{n+k-1} P_{k-2}, \\
Q_{k}=\alpha(n, k)\left(4 k(n+k) I-D^{2}\right) Q_{k-1}+(-1)^{k} D^{n+k-1} Q_{k-2} .
\end{array}\right.
$$

We see that $P_{k}$ and $Q_{k}$ are diagonal matrices. By setting $p_{k}=\operatorname{diag}\left(p_{k}^{1}, p_{k}^{2}, \ldots, p_{k}^{m}\right)$ and $q_{k}=\operatorname{diag}\left(q_{k}^{1}, q_{k}^{2}, \ldots, q_{k}^{m}\right)$, we obtain for each i where $1 \leq i \leq m$,

$$
\begin{gathered}
\left\{\begin{array}{l}
p_{-1}^{i}=1, p_{0}^{i}=\frac{\lambda^{n}}{2^{n} n !}, p_{1}^{i}=\frac{\lambda_{i}^{n}}{2^{n+1}(n+1) !} \\
q_{-1}^{i}=O, q_{0}^{i}=1, q_{1}^{i}=1,
\end{array}\right. \\
\left\{\begin{array}{l}
p_{2}^{i}=\frac{(n+2)}{2^{n}(n+1) !} \lambda_{i}^{n}\left(1-\lambda_{i}^{2}\right)\left((n+1)+\lambda_{i}^{2}\right), \\
q_{2}^{i}=\frac{1}{2^{n+1}(n+1) !}\left(2^{n+2}(n+2) !-2^{n+1}(n+1) ! \lambda_{i}^{2}-\lambda_{i}^{n+2}\right)
\end{array}\right.
\end{gathered}
$$

and for $k \geq 3$,

$$
\left\{\begin{array}{l}
p_{k}^{i}=\alpha(n, k)\left(4 k(n+k)-\lambda_{i}^{2}\right) p_{n-1}^{i}+(-1)^{k} \lambda^{n+k-1} p_{k-2}^{i} \\
q_{k}^{i}=\alpha(n, k)\left(4 k(n+k)-\lambda_{i}^{2}\right) q_{k-1}^{i}+(-1)^{k} \lambda^{n+k-1} q_{k-2}^{i}
\end{array}\right.
$$

By theorem 2, the convergent $\left(p_{n}^{i} / q_{n}^{i}\right)$ converges to $J_{n}\left(\lambda_{i}\right)$. It follows that $P_{n} / Q_{n}$ converges to the matrix $J_{n}(D)$, so that

$$
J_{n}(D)=\left[\frac{D^{n}}{2^{n} n !} ; \frac{\frac{-D^{n+2}}{2^{n+1}(n+1) !}}{I}, \frac{D^{2}}{2^{3}(n+2) I-D^{2}}, \frac{(-1)^{k} D^{n+k-1}}{\alpha(n, k)\left(4 k(n+k) I-D^{2}\right)}\right]_{k=3}^{+\infty}
$$

By proposition 2, we get

$$
\begin{aligned}
J_{n}(A) & =X\left[\frac{D^{n}}{2^{n} n !} ; \frac{\frac{-D^{n+2}}{2^{n+1}(n+1) !}}{I}, \frac{D^{2}}{2^{3}(n+2) I-D^{2}}, \frac{(-1)^{k} D^{n+k-1}}{\alpha(n, k)\left(4 k(n+k) I-D^{2}\right)}\right]_{k=3}^{+\infty} X^{-1} \\
& =\left[X \frac{D^{n}}{2^{n} n !} X^{-1} ; \frac{\frac{-D^{n+2} X^{-1}}{2^{n+1}(n+1) !}}{X^{-1}}, \frac{D^{n+2} X^{-1}}{2^{3}(n+2) I-D^{2}}, \frac{(-1)^{k} D^{n+k-1}}{\alpha(n, k)\left(4 k(n+k) I-D^{2}\right)}\right]_{k=3}^{+\infty} .
\end{aligned}
$$

Let us define the sequence $\left(X_{n}\right)_{k \geq-1}$ by

$$
\left\{\begin{array}{l}
X_{-1}=X_{0}=I \\
X_{k}=X \text { for all } k \geq 1
\end{array}\right.
$$

Then

$$
\left\{\begin{array}{l}
\frac{X_{1} B_{1} X_{-1}^{-1}}{X_{1} A_{1} X_{0}^{-1}}=\frac{\frac{-X D^{n+2} X^{-1}}{2^{n+1}(n+1) !}}{X X^{-1}}=\frac{\frac{-A^{n+2}}{2^{n+1}(n+1) !}}{I} \\
\frac{X_{2} B_{2} X_{0}^{-1}}{X_{2} A_{2} X_{1}^{-1}}=\frac{X D^{2} X^{-1}}{X\left(2^{3}(n+2) I-D^{2}\right) X^{-1}}=\frac{A^{2}}{2(n+2) I-A^{2}} .
\end{array}\right.
$$


For $k \geq 3$, we have

$$
\frac{X_{k} B_{k} X_{k-2}^{-1}}{X_{k} A X_{k-1}^{-1}}=\frac{(-1)^{k} A^{n+k-1}}{2^{n+2 k-4}(k-2) !(n+k-1) !\left(4 k(n+k) I-A^{2}\right)} .
$$

By applying the result of proposition 4 to the sequence $\left(X_{n}\right)_{n \geq-1}$, we finish the proof of theorem 2 .

\section{Acknowledgement}

The authors would like to express their sincere gratitude to the referee for a very careful reading of this paper and for all his/her insightful comments, which lead a number of improvements to this paper.

\section{References}

[1] T. Ando, Topics on operators inequalities, Ryukyu Univ. Lecture note Series 1, 1978.

[2] A.Cuyt, V.Brevik Petersen, Handbook of continued fractions for special functions, Springer (2007).

[3] F. R.Gantmacher. The Theory of Matrices, Vol. I. Chelsa, New York, Elsevier Science Publishers, (1992).

[4] V. Gen H.Golub and Charles F.Van Loan, Matrix Computations, Johns Hopking University Press, Baltimore, MD, USA, third edition (1996).

[5] T. L. HAYDEN, Continued fractions in Banach spaces, Rocky Mtn. J.Math., 4 (1974), pp. 367-369.

[6] A.N, Khovanski, The applications of continued fractions and their Generalisation to problemes in approximation theory,1963, Noordhoff, Groningen, The Netherlands (chap2).

[7] L.Lorentzen, H.Wadeland, Continued fractions with application, Elseiver Science Publishers, (1992).

[8] Gerard J. MURPHY, $C^{*}$-Algebras and operators theory, (1990), Academic press, INC Harcourt Brace Jovanovich, publishers.

[9] N. NEGOESCU, Convergence theorems on noncommutative continued fractions, Rev. Anal. Numér. Théorie Approx., 5 (1977), pp. $165-180$

[10] M.Raissouli, A.Kacha, Convergence for matrix continued fractions. Linear Algebra and its Applications, 320 (2000), pp. 115-129. 